\title{
Estrategias de afrontamiento a las reacciones emocionales de los padres de niños con fisura labial, palatina y labiopalatina
}

\author{
Badillo-Prudencio Laura1, Diaz-Urbina Angie'1, Inca-Serna María1, Bolaños-Gil Hilda²
}

\section{RESUMEN}

Objetivo: determinar las estrategias de afrontamiento a las reacciones emocionales de las madres de niños con labio leporino, fisura del paladar y fisura del paladar con labio leporino de 0 a 2 años en el Instituto Nacional de Salud del Niño de Lima-Perú en el año 2012. Material y métodos: enfoque cuantitativo de tipo descriptivo, se realizó en el Instituto Nacional de Salud del Niño ubicado en el distrito de Breña, en el servicio Consultorios Externos de Cirugía plástica. Para la recolección de los datos se elaboró un cuestionario subdividido en dos partes: datos sociodemográficos, y 26 preguntas divididas en trece estrategias relacionadas a las dimensiones de afrontamiento, enfocadas al problema, a la emoción y evitación del problema. Dicho instrumento fue validado por juicio de expertos y prueba piloto. La población estuvo conformada por 60 madres de familia. Resultados: los resultados mostraron que las estrategias enfocadas en el problema obtuvieron una media de 3,7; mientras que las estrategias enfocadas en la emoción una media de 3,11 y, por último, las estrategias enfocadas en la evitación del problema una media de 3,13. Conclusiones: un porcentaje mayor de madres presentó un uso de estrategias enfocadas en el problema, dentro de ellas el afrontamiento activo y la reinterpretación positiva fueron las más frecuentes. Las estrategias enfocadas a la emoción y las estrategias enfocadas en la evitación del problema obtuvieron resultados similares, dentro de ellas las más usadas fueron: la estrategia de reinterpretación positiva - crecimiento; aceptación- resignación, enfocadas a la emoción; en el grupo de evitación del problema fueron las estrategias de desentendimiento mental y conductual.

Palabras clave: labio leporino, fisura del paladar, estrategias. Perú. (Fuente DeCs BIREME).

\section{Strategies of confrontation to the emotional reactions of the parents of children with fissure lip, court, lip and court}

\begin{abstract}
Objetive: To determine the coping strategies to emotional reactions to mothers of children with harelip, cleft palate and cleft palate with harelip from 0 to 2 years old at the National Institute of Child Health in Lima, Peru in 2012. Material and Methods: This is descriptive and quantitative approach. The study was realized at the National Institute of Child Health in External Doctor Service of Plastic Surgery located in Breña. In order to collect the information. It was elaborated a questionnaire. It was two parts: social -demographic questions and 26 questions of the coping strategies focused to problem, emotion and the avoidance. This questionnaire was validated by expert's judgment and a pilot testing. The population was composed by 60 mothers. Results: In the coping strategies focused to problem, emotion and the avoidance obtained 3.7, 3.13, and 3.11 of half respectively. Conclusion: A higher percentage of mothers
\end{abstract}

Estudiante de la Facultad de Enfermería. Universidad Peruana Cayetano Heredia. Lima - Perú.

Magister en Enfermeria. Esp. en Pediatría. Docente auxiliar de la Universidad Peruana Cayetano Heredia. Lima - Perú. 
presented a use of strategies focused to problem inside of them the active coping and positive reinterpretation were the most frequent. The strategies focused to emotion and avoidant obtained similar results inside of them the most used were positive reinterpretation strategy - growth. In the focused on the emotion and the avoidant the strategy acceptanceresignation and release emotions were the most used respectively. The strategies: Mental disengagement and behavioral disengagement were the most used in the group focused to avoidant.

Key words: Cleft lip, cleft palate, strategies. Peru. (Source DeCs BIREME).

\section{INTRODUCCIÓN}

El trabajo es sobre las estrategias de afrontamiento a las reacciones emocionales de las madres de niños de 0 a 2 años con labio leporino, fisura del paladar y fisura del paladar con labio leporino, que ayudará a que el personal de enfermería encargado del cuidado del paciente pediátrico con dicha malformación, contribuya en el proceso de aceptación de las madres hacia su hijo, orientándolos sobre las estrategias de afrontamiento para un mejor manejo de las diferentes reacciones emocionales generadas por la malformación congénita.

En el país, cada año 2500 niños nacen con labio leporino, fisura del paladar y fisura del paladar con labio leporino; el 25\% de estos niños padecen de fisura del paladar, 25\% de labio leporino, y el 50\%, de ambos. Esta malformación congénita es más común en los niños que en las niñas y ocurre con más frecuencia en los países en vía de desarrollo (1).

Para todos los profesionales de la salud dedicados a la atención de los niños, es muy importante el trabajo con las madres ya que estas son una constante en la vida de cualquier niño, la familia es uno de los factores que más influye en su vida futura, aún más si tienen alguna alteración en su desarrollo (2).

Cuando se presenta una malformación congénita en un hijo, las madres desarrollan en muchas ocasiones sentimientos de ansiedad, enojo, cólera y odio que se manifiestan de forma inconsciente o consciente. Este suceso es el más difícil de afrontar y cada persona tiene actitudes diferentes que pueden ir desde la negación de esta hasta la aceptación. Las madres del niño con labio leporino, fisura del paladar y fisura del paladar con labio leporino, pueden tener sentimientos de culpa, frustración, castigo o de injusticia, de acuerdo con sus patrones socioculturales y religiosos.

La búsqueda de una respuesta a la malformación congé- nita de su hijo, es una pregunta continua y desesperada de cada uno de los padres. La necesidad de mitigar el dolor de la realidad y retomar la vida misma, es lo que motiva el ofrecer una alternativa socio-terapéutica para avanzar en el proceso de aceptación.

Desde esta óptica, el brindar cuidado a los seres humanos con dolor, principalmente el de las madres ante el nacimiento de su hijo con labio leporino, fisura del paladar y fisura del paladar con labio leporino, se convierte en un elemento central de la labor de enfermería, debido al impacto generado por el evento, donde la vida se desmorona (3).

Algunos de los aspectos considerados como determinantes en esta experiencia son las sistemáticas frustraciones de las madres en el proceso de alimentación del bebé (4), la ansiedad que les produce su defecto facial, las reacciones de los familiares y amigos, la preocupación por el desarrollo emocional de su bebé entre otros.

Las reacciones son diferentes en cada una de las diversidades de la enfermedad. En la fisura del paladar con labio leporino bilateral y unilateral se genera un mayor impacto emocional en las madres, ya que el defecto facial es más evidente y las reacciones de los familiares más notorias. Esto conlleva a algunas madres a aislarse, esconder al niño por miedo a las reacciones de la gente, así como dificulta mas su alimentación.

En estos casos la alimentación del bebé se hace complicada para las madres. Las alteraciones anatómicas y funcionales del recién nacido con fisura del paladar con labio leporino dificultan la lactancia materna, facilitan las infecciones respiratorias y la erosión de la mucosa oral expuesta, sin protección de la piel. Generan una disminución en la ganancia de peso y, por otra parte el número de operaciones a realizar en el pequeño son mayores.

La fisura del paladar no es tan perceptible debido a que se encuentra dentro de la boca. La preocupación más in- 
mediata para un bebé que tiene la fisura del paladar es la alimentación. Para los niños que tienen la fisura del paladar, la deglución es difícil a causa de su malformación. Los niños que tienen sólo labio leporino, normalmente no tienen dificultades de alimentación, los problemas son más que todo estéticos (5).

Recientes investigaciones identifican que mientras más dificultades presente el niño, las madres evidencian estrategias de afrontamiento enfocadas a las emociones, en cambio cuando los síntomas son más leves hacen uso de estrategias de afrontamiento enfocadas al problema. Por ello, es importante elaborar estrategias de intervención en donde se busque empoderar a estas madres en su rol como tal y ayudarlas a percatarse de las herramientas tanto emocionales como cognitivas con las que cuentan (6).

\section{MATERIAL Y MÉTODOS}

Según el problema y los objetivos de investigación se utilizó el tipo de estudio descriptivo con un enfoque cuantitativo.

El estudio se realizó en el Instituto Nacional de Salud del Niño ubicado en la Av. Brasil 600 Breña-Lima, consultorio externo de Cirugía Plástica, el cual es un área especializada en la atención de niños con labio leporino, fisura del paladar y fisura del paladar con labio leporino.

La población estuvo conformada por un total de 60 madres cuyos hijos presentan labio leporino, fisura del paladar y fisura del paladar con labio leporino de 0 a 2 años atendidos en el INSN.

Se aplicaron los criterios de inclusión; madres mayores de 18 años de edad de niños con labio leporino, fisura del paladar y fisura del paladar con labio leporino de 0 a 2 años y madres que acepten participar en la realización del estudio de investigación, Como criterios de exclusión, madres de niños con labio leporino, fisura del paladar y fisura del paladar con labio leporino de 0 a 2 años con alteración psiquiátrica.

Para el estudio se tomó el total de la población.

La técnica utilizada fue la encuesta y se utilizó como instrumento el cuestionario COPE (Cuestionario de Estrategias de Afrontamiento) de Carver, Scheir y Weintraub fue creado en 1989 (7).
La primera parte contiene datos sociodemográficos de los participantes con la finalidad de obtener información sobre algunos datos relevantes de la muestra, para así utilizarlas en el enriquecimiento del análisis de las variables de estudio.

La segunda parte consta de 26 ítems que abarca las trece estrategias de afrontamiento (dos ítems por cada una) con respuesta tipo Likert de cuatro alternativas de respuesta. Los ítems 1 al 17, 21, 22 tienen como alternativas de respuestas: casi nunca, rara vez, algunas veces, siempre con un puntaje de calificación de 1, 2, 3 y 4 respectivamente debido a que su uso es considerado favorable.

Los ítems 18, 19, 20, 23, 24, 25, 26 tienen como alternativas de respuestas casi nunca, rara vez, algunas veces, siempre con un puntaje de calificación de 4, 3, 2 y 1 respectivamente debido a que su uso es considerado desfavorable.

Los indicadores fueron representados numéricamente mediante la escala tipo Likert.

Para dar validez de contenido, se sometió a juicio de expertos, el cual estuvo constituido por siete profesionales de la salud del Instituto Nacional de Salud del Niño (médico, enfermera, tecnólogo, psicólogo); la confiabilidad se obtuvo del coeficiente alfa de Cronbach, el cual fue superior a $0,6(8)$. Esta se obtuvo mediante la prueba piloto que se realizó con 15 madres de niños con labio leporino, fisura del paladar y fisura del paladar con labio leporino del servicio de consultorio externo de cirugía plástica del INSN.

Para la recolección de datos, se coordinó con el Médico Jefe del servicio de consultorios externos de Cirugía Plástica los días que se irían a realizar las encuestas a las madres. Momentos previos al desarrollo del cuestionario se informó a las madres sobre los objetivos, la forma de participación y los beneficios del estudio (contenido educativo), en un lenguaje claro y sencillo. Posterior a ello se hizo entrega de la hoja de consentimiento informado para obtener la firma con la que autorizaban su participación. Se entregó la hoja del cuestionario a las madres y se les explicaba la dinámica de llenado.

Los datos fueron codificados e ingresados a una base de datos creado en el programa estadístico SPSS (Paquete Estadístico para las Ciencias Sociales) versión 15.0, para su posterior análisis. 


\section{RESULTADOS}

Tabla 1. Estrategias de afrontamiento a las reacciones emocionales de las madres de niños con labio leporino, fisura del paladar y fisura del paladar con labio leporino de 0 a 2 años enfocadas en el problema, 2012

\begin{tabular}{lrrrrrrrr}
\hline $\begin{array}{l}\text { Estrate gias de afrontamiento } \\
\text { enfocad as en el problema }\end{array}$ & \multicolumn{2}{c}{ Bajo } & \multicolumn{2}{c}{ Medio } & \multicolumn{2}{c}{ Alto } & \multicolumn{2}{c}{ Total } \\
\hline Afrontamiento activo & $\mathbf{\%}$ & $\mathbf{N}$ & $\mathbf{\%}$ & $\mathbf{N}$ & $\mathbf{\%}$ & $\mathbf{N}$ & $\%$ \\
Planificación & 4 & 1,7 & 5 & 8,3 & 54 & 90,0 & 60 & 100 \\
Supresión de otras actividades & 11 & 18,3 & 14 & 23,3 & 42 & 70,0 & 60 & 100 \\
Postergación del afrontamiento & 12 & 20,0 & 15 & 21,7 & 36 & 60,0 & 60 & 100 \\
$\begin{array}{l}\text { Búsqueda de apoyo social por } \\
\text { razones instrumentales }\end{array}$ & 11 & 18,4 & 20 & 33,3 & 29 & 48,3 & 60 & 100 \\
\hline
\end{tabular}

Tabla 2. Estrategias de afrontamiento a las reacciones emocionales de las madres de niños con labio leporino, fisura del paladar y fisura del paladar con labio leporino de 0 a 2 años enfocadas en la emoción, 2012

\begin{tabular}{|c|c|c|c|c|c|c|c|c|}
\hline \multirow{2}{*}{$\begin{array}{l}\text { Estrategias de afrontamiento } \\
\text { enfocadas en la emoción }\end{array}$} & \multicolumn{2}{|c|}{ Bajo } & \multicolumn{2}{|c|}{ Medio } & \multicolumn{2}{|c|}{ Alto } & \multicolumn{2}{|c|}{ Total } \\
\hline & $\mathbf{N}$ & $\%$ & $\mathbf{N}$ & $\%$ & $\mathbf{N}$ & $\%$ & $\mathbf{N}$ & $\%$ \\
\hline $\begin{array}{l}\text { Búsqueda de apoyo social por } \\
\text { razones emocionales }\end{array}$ & 13 & 21,6 & 30 & 50,0 & 17 & 28,4 & 60 & 100 \\
\hline Aceptación- resignación & 11 & 18,3 & 7 & 11,7 & 42 & 70,0 & 60 & 100 \\
\hline Acudir a la religión & 22 & 36,7 & 35 & 58,3 & 3 & 5,0 & 60 & 100 \\
\hline $\begin{array}{l}\text { Reinterpretación positiva y } \\
\text { crecimiento }\end{array}$ & 9 & 15,0 & 10 & 16,7 & 44 & 73,3 & 60 & 100 \\
\hline Negación & 18 & 30,0 & 11 & 18,3 & 31 & 51,7 & 60 & 100 \\
\hline
\end{tabular}

Tabla 3. Estrategias de afrontamiento a las reacciones emocionales de las madres de niños con labio leporino, fisura del paladar y fisura del paladar con labio leporino de 0 a 2 años enfocadas en la evitación del problema, 2012

\begin{tabular}{|c|c|c|c|c|c|c|c|c|}
\hline \multirow{2}{*}{$\begin{array}{l}\text { Estrategias de afrontamiento } \\
\text { enfocadas en la evitación del } \\
\text { problema }\end{array}$} & \multicolumn{2}{|c|}{ Bajo } & \multicolumn{2}{|c|}{ Medio } & \multicolumn{2}{|c|}{ Alto } & \multicolumn{2}{|c|}{ Total } \\
\hline & $\mathbf{N}$ & $\%$ & $\mathbf{N}$ & $\%$ & $\mathbf{N}$ & $\%$ & $\mathbf{N}$ & $\%$ \\
\hline Enfocar y liberar emociones & 24 & 40,0 & 20 & 33,3 & 16 & 26,7 & 60 & 100 \\
\hline Desentendimiento conductual & 13 & 21,6 & 12 & 20,0 & 35 & 58,3 & 60 & 100 \\
\hline Desentendimiento mental & 12 & 20,0 & 7 & 11,7 & 41 & 68,3 & 60 & 100 \\
\hline
\end{tabular}

\section{DISCUSIÓN}

Los resultados muestran que las estrategias más usadas en el grupo de estrategias enfocadas en el problema fueron: afrontamiento activo (90\%) y planificación (70\%), la menos usada fue búsqueda de apoyo social por razones instrumentales con un 48, $3 \%$ (Tabla 1).

En el estudio de Baker, el afrontamiento dominante por los padres reportaban un enfoque orientado a la búsqueda de apoyo, resolución de problemas, el análisis lógico y positivo evaluación, en lugar de un enfoque orientado a la evitación cognitiva, aceptación, las recompensas alternativas, descarga emocional (9).

Una vez que las madres superan el shock inicial reflexionan sobre este acontecimiento y optan por la búsqueda de la solución del problema enfocando su atención a la información, apoyo médico, que mejorará la calidad de vida del niño. Es así que las madres que reciben información y asesoramiento por parte de los profesionales de la salud acerca del problema de su hijo, tienden a tomarlo 
de una manera más positiva en comparación con los que no se le informan o desconocen el diagnóstico de su hijo. $\mathrm{Si}$ estos servicios son adecuados, pueden constituir un verdadero factor de protección y de resistencia para afrontar las demandas internas y externas relacionadas con la enfermedad de su hijo (10).

Los resultados muestran que las estrategias más usadas en el grupo de estrategias enfocadas en la emoción fueron: reinterpretación positiva, crecimiento $(73,3 \%)$; aceptación-resignación (70\%); negación con un 51,7\%, y la menos usada fue acudir a la religión con un 5\% (Tabla 2).

La estrategia de acudir a la religión es la tendencia a volcarse en la religión en situaciones de estrés con la intención de reducir la tensión existente. Este tipo de estrategia fue utilizada en menor medida en las madres de nuestro estudio, debido a que la enfermedad de sus hijos tiene buen pronóstico y que mediante tratamiento quirúrgico y de las demás especialidades el niño puede volver a tener su vida normal (11).

Así mismo en el estudio de Limiñana $\mathrm{R}$, se refiere que los padres obtienen un estilo preferente de estrategia de afrontamiento enfocadas al problema (4). Los padres no informan del uso de otras estrategias de afrontamiento enfocadas a la emoción. La estrategia de reevaluación positiva, obtuvo una media de 5,53, siendo la más usada en el grupo de enfocadas a la emoción. Así, en estos estudios la estrategia enfocada a la emoción es la menos utilizada, si bien el impacto que causa la presencia de un niño con labio leporino, fisura del paladar y fisura del paladar con labio leporino es mayor en un inicio, las madres utilizan esta estrategia para conservar la esperanza y tratar de aceptar de una mejor forma este suceso.

El uso de la estrategia enfocada a la emoción es considerada como positivo debido a que permite manejar emociones negativas e intensas como la ansiedad y cólera que pueden estar experimentando las madres de los niños con fisura del paladar con labio leporino. Por ello, este estilo es particularmente útil cuando poco o nada puede hacerse en el momento para resolver definitivamente un evento ya que ayuda a mantener la moral y el sentido de esperanza (12).

Los resultados muestran que las estrategias más usadas en el grupo de estrategias enfocadas en la evitación del problema fueron: desentendimiento mental y conductual con un $68,3 \%$ y $58,3 \%$ respectivamente y la menos usada enfocar y liberar emociones con un 26,7\% (Tabla $3)$.
Así Limiñana (4) en su estudio refiere que uso de estrategias enfocadas en la evitación del problema parece ser muy escaso teniendo una media de 1,55.

En el estudio de Evelyn Lagos (13), en relación a las estrategias de afrontamiento, se puede observar que los padres manifestaron mayor uso de las estrategias: reinterpretación positiva, autocontrol y resolución planificada, siendo las menos utilizadas: aceptación de la responsabilidad, confrontación y distanciamiento.

Cuando las madres emplean estrategias de evitación, como el desentendimiento conductual y la negación, se ve afectado su rol de madres, por lo tanto, interfieren en la dinámica que establece con su hijo. Ello implica que las madres que buscan evadir las complicaciones en la crianza de un niño con labio leporino, fisura del paladar y fisura del paladar con labio leporino, para focalizarse en otras actividades, no logran hacer frente a los problemas, por consiguiente estos persisten e inclusive puede generar en las madres depresión y aislamiento (14). Sin embargo, en otras investigaciones consideran que el emplear estrategias de afrontamiento enfocadas en la evitación del problema sirve como protección para las madres, en especial cuando los síntomas de los niños son severos. Ante este hecho las estrategias enfocadas en la evitación del problema parecen útiles en la medida en que reducen el estrés y evitan que la ansiedad llegue a ser perturbadora (15).

Cuando las madres se enteran de la malformación de su hijo sobreviene una serie de reacciones emocionales como: ira, tristeza e impotencia, que deben ser consideradas como respuestas normales ante una agente estresor, que es el saber que su hijo tiene un defecto físico y todo lo que ello implica, y que cada persona maneja de modo diferente estas reacciones, en donde algunos buscan huir, escapar del problema, ya que esto les genera un mayor alivio (16). Estas reacciones no deben ser tomadas de manera negativa, debido a que en estas circunstancias las madres requiere más apoyo para sobrellevar ese problema y es nuestro deber encaminarlo en bienestar del hijo mediante el trabajo interdisciplinario que incluya orientación psicológica para las madres.

\section{REFERENCIAS BIBLIOGRÁFICAS}

1. Asociación Operación Sonrisa. Patología labio leporino [Internet] 2010. [citado 16 May 2011]. Disponible en: http://www.operacionsonrisa.org.pe/patologias.html

2. Jiménez F, Loscertales M, Martínez A, Barbancho M, Lanzarote D, Nieto M. Padres de recién nacidos ingresados en UCIN, impacto emocional y familiar. 
Vox Pediátrica [Internet] 2003 noviembre. [citado 25 Mar 2011] 27-33 Disponible en: http://www.sld.cu/ galerias/pdf/sitios/rehabilitaciontemprana/ padres_de_recien_nacidos_en_ucin.pdf

3. Gutiérrez A. Manejo de duelo en las madres y padres que pierden a su hijo o hija de forma inesperada [tesis] [Internet]. Costa Rica: Universidad de Costa Rica. 2009. [citado 28 Mar 2011]. Disponible en: http:// www.ts.ucr.ac.cr/binarios/tfgmae/tfg-m-2009-01.pdf

4. Limiñana R, Corbalán Berná F, Patrón R. Afrontamiento y adaptación psicológica en padres de niños con fisura palatina. Anales de psicología [Internet] 2007 julio [citado 26 Nov 2012]; 23(2). Disponible en: http://revistas.um.es/analesps/article/ view/22431

5. Protocolo Auge fisura labio palatina para niños [Internet]. Chile MINSA Abril 2004. [citado 4 Abr 2012]. Disponible en: http://www.epidemiologia.anm. edu.ar/fisura_palatina/pdf/Protocolo\%20ChileFlap\% 20ni\%C3\%B1os.pdf

6. Pineda G. Estrés parental y estilos de afrontamiento en padres de niños con trastornos el espectro autista. [tesis]. Lima: Pontifica Universidad Católica del Perú; 2012. [citado 25 Ago 2012]. Disponible en: http:// tesis.pucp.edu.pe/repositorio/handle/123456789/1659

7. Carver CS, Scheier MF, Weintraub JK. Assessing coping strategies: a theoretically based approach. Journal of Personality and Social Psychology 1989. 56:267-883.

8. Hernández R, Fernández C, Baptista L. Metodología de la Investigación. 4 ed. Mc Graw Hill. Interamericana. México; 2006.

9. Baker R, Owens J, Stern, Willmot D. Estrategias de afrontamiento y apoyo social en el impacto familiar de labio leporino y el paladar y el ajuste de los padres y la angustia psicológica. 2009 may. United States [citado 28 Abr 2012]. Disponible en: http://www. cpcjournal.org/doi/abs/10.1597/08-075.1

10. Guevara Y., González Edith. Las familias ante la discapacidad. Rev. Electrónica de Psicología Iztacala 15, (3), 2012. [citado 5 Feb 2013] Disponible en: http:/ /www.iztacala.unam.mx/carreras/psicologia/psiclin/ vol15num3/Vol15No3Art13.pdf

11. Fernández E Ma. 2009. Estrés percibido, estrategias de afrontamiento y sentido de coherencia en estudiantes de enfermería: su asociación con salud psicológica y estabilidad emocional. [tesis doctoral]. [citado 25 Feb 2013]. Disponible en: https:// buleria.unileon.es/bitstream/handle/10612/902/ 2009FERN\%C3\% 81NDEZ\%20MART \%C3\% 8DNEZ,\%20MAR\%C3\%8DA\%20ELENA.pdf? sequence $=1$

12. Cassaretto M. Relaciones entre la personalidad y el afrontamiento en estudiantes preuniversitarios. Revista vanguardia psicológica. Lima. 2010 [citado 25 Oct 2012] Disponible en: http://umb.edu.co:82/ revp/index.php/rvp/article/viewfile/28/9

13. Lagos E. Estrategias de afrontamiento de padres de niños con secuelas de quemaduras visibles. Rev. de psicología. Chile. 2004. [Citado 8 Oct 2012]. Disponible en: http://redalyc.uaemex.mx/src/inicio/ forazardescarga archivo.jsp ?cvrev $=264 \&$ cvart $=26413205 \&$ nombre $=$ estrategias $\% 20 \mathrm{de} \% 20$ afrontamiento $\% 20 \mathrm{de} \% 20$ padres $\% 20 \mathrm{de} \% 20 \mathrm{ni} \%$ f1 os $\% 20$ con $\% 20$ secuelas $\% 20$ de $\% 20$ quemaduras $\%$ 20visibles

14. Protocolo Auge fisura labio palatina para niños abril 2004. [citado 4 Abr 2012]. Disponible en: http:// www.epidemiologia.anm.edu.ar/fisura_palatina/pdf/ Protocolo\%20Chile-Flap\%20ni\%C3\%B1os.pdf

15. Paredes M, Cassaretto R. Afrontamiento y soporte social en un grupo de pacientes con insuficiencia renal crónica terminal en un hospital del seguro social peruano. [tesis]. Perú. Pontificia Universidad Católica del Perú. [citado 25 Feb 2013].Disponible en: http:// es.scribd.com/doc/47931677/to-y-Soporte-Social-enUn-Grupo-de-Pacientes-Con-Insuficiencia-RenalCrnica-Terminal

16. Fuchs E, Leichner M. Afrontamiento en madres durante el tratamiento de Fisura Labio Alveolo Palatina (FLAP) de sus hijos [Internet]. [tesis]. Argentina. Pontifica Universidad Católica Argentina [citado 4 Feb 2013]. Disponible en:http://biblioteca digital.uca.edu.ar/repositorio/tesis/afrontamientomadres-durante-tratamiento-flap.pdf

\section{Correspondencia}

Hilda Bolaños Gil

Miguel Baquero 251, Lima.

Telefóno: 4314731 - 4244731

Correo electrónico: hilda.bolanos@upch.pe

Forma de citar este artículo: Badillo-Prudencio L, Diaz-Urbina A, Inca-Serna M, Bolaños-Gil H. Estrategias de afrontamiento a las reacciones emocionales de los padres de niños con fisura labial, palatina y labiopalatina. Rev enferm Herediana. 2013;6(1):12-17. 